\title{
Using Heart Failure with Preserved Ejection Fraction to Understand an 'Omics Approach for Evaluating Vascular Dysfunction and Cardiovascular Disease
}

\author{
Rosita Zakeri ${ }^{1}$, Catalina Vallejo-Giraldo ${ }^{2}$, K. Efua Taylor ${ }^{2}$, Virginia M. Miller²,3* and Niccole S. Schaible ${ }^{2}$
}

${ }^{1}$ Division of Cardiovascular Diseases, Department of Internal Medicine, Mayo Clinic College of Medicine, Rochester, MN 55905 ${ }^{2}$ Physiology and Biomedical Engineering, Department of Internal Medicine, Mayo Clinic College of Medicine, Rochester, MN 55905 ${ }^{3}$ Surgery, Department of Internal Medicine, Mayo Clinic College of Medicine, Rochester, MN 55905

\begin{abstract}
Regulation of vascular tone is complex due to the presence of redundant mechanisms operating over multiple spatial domains (cell, tissue and organ) and influenced by various confounding factors, such as sex, age and time. As a result, the task of identifying the underlying mechanisms of vascular dysfunction associated with complex cardiovascular diseases, like heart failure with preserved ejection fraction (HFpEF), remains under explored. 'Omics research strategies are proposed to be an effective way to navigate complicated physiology. In this review an 'omics approach is applied to the spatial domains of HFpEF using a mechanistic "physiological map" of factors known to be associated with vascular (dys) function. The result is intended to generate hypotheses, foster robust study design, and highlight the necessity for collaborative, interdisciplinary approaches to conduct 'omics research, which in turn is expected to facilitate the development of efficacious therapies for cardiovascular diseases such as HFpEF, where etiologies are unclear and evidence-based therapeutic strategies lacking.
\end{abstract}

Keywords: Gene expression; Proteomics; Cardiovascular disease; Heart failure with preserved ejection fraction; Hypertension; Physiology; Vasculomics

\section{Introduction}

An example of a nexus of integrated control within the body is regulation of vascular tone. The inherent complexity in analyzing vascular function lies not only in the fact that control is mediated, in part, by the dynamic interaction of the autonomic nervous system with local biochemical and physical forces (hemodynamics), spanning spatial domains and time scales, but ultimately because the sum total of all aspects of the vascular system are integrated with other control systems and influenced by genetic, developmental and environmental factors. For example, redundant negative feedback systems operate through neurohumoral mechanisms to regulate blood volume and blood pressure, but the relative influence of each will differ depending upon the anatomical location of a particular circulatory bed (i.e. systemic, pulmonary, coronary, etc.) as well as additional confounding factors such as sex and age, defined in part by reproductive status (sexually immature, reproductively competent or senescent). Study of this type of complexity is classically known as "physiology" and often investigated through hypothesis-driven experimental protocols addressing narrowly defined foci of interest. However, due to technological advances over the last decade, a new field has emerged to study much of the same topics but utilizing the wealth of burgeoning 'omics data including genomics, proteomics, and metabolomics, enabling a more holistic approach to the study of biological systems [1] .

The vascular system is a particularly attractive candidate for an 'omics style investigation both in health and disease. Initial forays have generated new insights into thrombosis and hemostasis, atherosclerosis, angiogenesis and vascular remodeling [2]. There are concerns about the proper use and interpretation of 'omics results given the inherent biases associated with high throughput data obtained from differing sources and collection techniques. However, meticulous attention to study design can ameliorate this problem, and present 'omics-technologies as an attractive and complementary way to examine cardiovascular physiology and disease. When considering the control of vascular tone, for instance, aging is associated with increased pulmonary vascular pressures and systemic vascular stiffening contributing to hypertension. For some patients these physiological traits predispose to heart failure,

\begin{tabular}{|l|l|l|l|}
\hline Baseline variables & $\begin{array}{l}\text { HFrEF } \\
\text { (LVEF<40\%; } \\
\text { n=1540) }\end{array}$ & $\begin{array}{l}\text { HFpEF } \\
\text { (LVEF>50\%; } \\
\text { n=880) }\end{array}$ & p-value \\
\hline Mean LVEF, \% & 25.9 & 62.4 & $<0.001$ \\
\hline Age, y & $71.8 \pm 12$ & $75.4 \pm 11.51$ & $<0.001$ \\
\hline Female (\%) & 37.4 & 65.7 & $<0.001$ \\
\hline Coronary artery disease (\%) & 48.7 & 35.5 & $<0.001$ \\
\hline Angina (\%) & 28.0 & 22.8 & $<0.005$ \\
\hline Prior myocardial infarction (\%) & 39 & 16.6 & $<0.001$ \\
\hline Prior CABG (\%) & 12.9 & 5.8 & $<0.001$ \\
\hline Hypertension (\%) & 84 & 91 & $<0.001$ \\
\hline Diabetes (\%) & 38.9 & 31.7 & $<0.001$ \\
\hline Atrial fibrillation (\%) & 23.6 & 31.8 & $<0.001$ \\
\hline COPD (\%) & 13.2 & 17.7 & $<0.002$ \\
\hline Hemoglobin $<10 \mathrm{~g} / \mathrm{dL}$ & 9.9 & 21.1 & $<0.001$ \\
\hline Systolic BP, $\mathrm{mmHg}$ & 146 & 156 & $<0.001$ \\
\hline LVF, & & & \\
\hline
\end{tabular}

LVEF, left ventricular ejection fraction; CABG, coronary artery bypass grafting; COPD, chronic obstructive pulmonary disease.

Table 1: Characteristics of patients with heart failure and reduced ejection fraction versus heart failure with preserved ejection fraction. Adapted from Bhatia et al. [5].

${ }^{*}$ Corresponding author: Virginia M. Miller, Physiology and Biomedica Engineering, Surgery, Department of Internal Medicine, Mayo Clinic College of Medicine, Rochester, MN 55905, Tel: 507-284-2290; Fax: 507-266-2233 E-mail: miller.virginia@mayo.edu

Received July 29, 2011; Accepted August 31, 2011; Published September 20, 2011

Citation: Zakeri R, Vallejo-Giraldo C, Taylor KE, Miller VM, Schaible NS (2011) Using Heart Failure with Preserved Ejection Fraction to Understand an 'Omics Approach for Evaluating Vascular Dysfunction and Cardiovascular Disease. Neurol Neurophysiol S1. doi:10.4172/2155-9562.S1-006

Copyright: (c) 2011 Zakeri R, et al. This is an open-access article distributed unde the terms of the Creative Commons Attribution License, which permits unrestricted use, distribution, and reproduction in any medium, provided the original author and source are credited. 
Citation: Zakeri R, Vallejo-Giraldo C, Taylor KE, Miller VM, Schaible NS (2011) Using Heart Failure with Preserved Ejection Fraction to Understand an 'Omics Approach for Evaluating Vascular Dysfunction and Cardiovascular Disease. J Neurol Neurophysiol S1. doi:10.4172/2155-9562. S1-006

Page 2 of 10

and of recent interest, the syndrome of heart failure with preserved ejection fraction (HFpEF). HFpEF is a complex syndrome whose pathophysiology remains unclear and therapeutic strategies undefined.
In addition there is an unexplained female predominance, which has received little attention in experimental work to date. These unknowns suggest HFpEF is a potentially fruitful candidate for an 'omics-style

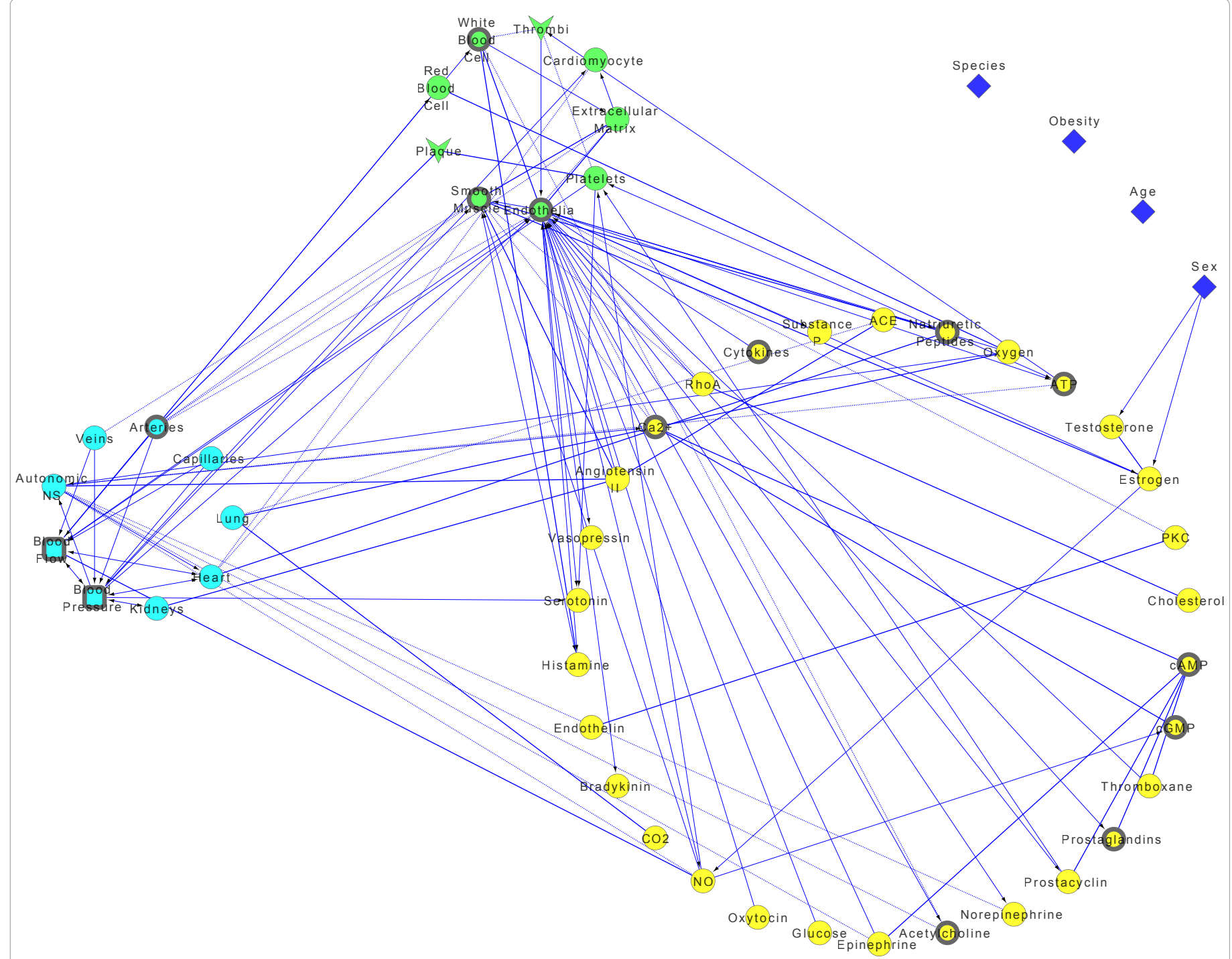

Figure 1: Vasculomic Network: cardiovascular physiology map for omics study design.

Key components of cardiovascular physiology are represented as nodes: cyan nodes $=$ organ-level domain, green nodes $=$ cellular domain, yellow nodes $=$ molecular domain, dark-blue nodes = organism domain. Nodes with a thick border indicate heterogeneity. The interactions between components are displayed as either arrows, which indicate cause-effect relationships, or dotted lines, which indicate associations. This figure was created using freely available software, Cytoscape (cytoscape. org), version 2.8.1 [75]. The network structure was created by N. Schaible based on "Vascular Pharmacology" (Ch. 5) by O'Rourke, Vanhoutte, and Miller [76].

This "physiological map" displays the primary components involved in vascular physiology which are represented as nodes, color-coded and grouped according to spatial domain. Note that the picture that emerges from a high-level inspection of the map is one of multiple levels of feedback between molecular, cellular, and organ-level operation. The relationships between components are represented with either solid arrows (cause/effect) or dotted lines (association). Therefore each line connecting a pair of nodes represents distinct physiological purpose. For example, a dotted-line connecting "cardiomyocytes" with "heart" is based upon the knowledge that the cardiomyocytes are constituents of the heart organ. Cardiomyocytes have a singular cellular identity, but also are collectively associated with the heart organ. An arrow in the physiological map, on the other hand, indicates a cause-and-effect relationship. For example, a unidirectional arrow from "blood flow" (organ domain) to "endothelial cell" (cell domain) reflects the understanding that endothelial cells are sensitive to shear stress which is attributed to blood flow. Even without concern of the identity of nodes and lines, a key feature easily visualized on this map, is the intimate interconnectedness across all spatial domains - which are certainly not unidirectional from gene to organ.

Note also that some nodes are more heavily interconnected than others; the nodes which have the most connections are the endothelial cells and the smooth muscle. This is because these two components are major integrators of vascular control. Vascular smooth muscle, by contracting or relaxing, has the capacity to significantly impact pressure/flow responses within the vasculature and as a result plays the important role as main effector of cardiovascular control. Smooth muscle, on its own, is sensitive to "external stimuli" provided by the autonomic nervous system. However, smooth muscle also receives input from endothelial cells, also a hub of control. The endothelial cells receive many inputs from the local environment, such as hormones, and respond to these cues mainly by affecting the contractile state of the smooth muscle. Also, the processing of input to output occurs in the molecular domain which involve many intermediates - most notably calcium ions. The central role of the smooth muscle and endothelial cells is another key point illustrated in the diagram. 
Citation: Zakeri R, Vallejo-Giraldo C, Taylor KE, Miller VM, Schaible NS (2011) Using Heart Failure with Preserved Ejection Fraction to Understand an 'Omics Approach for Evaluating Vascular Dysfunction and Cardiovascular Disease. J Neurol Neurophysiol S1. doi:10.4172/2155-9562. S1-006

investigation. In this review, pertinent physiological pathways thought to be related to the etiology of HFpEF will be used to illustrate the relative merits and important design considerations for the successful and vigilant application of 'omics-techniques to the study of vascular dysfunction and cardiovascular disease, also known as vasculomics. In addition this review presents a unique focus on experimental work delineating proposed molecular mechanisms underlying HFpEF, and particularly the potential use of 'omics to clarify poorly understood influences, such as the sex-disparity of this disease.

\section{HFpEF: A candidate for vasculomics research}

Heart failure is a major public health concern, affecting more than 5 million people in the United States, including 10 per 1000 of the population over age 65 [3]. Approximately half of all patients with chronic heart failure have preserved rather than reduced left ventricular ejection fraction (LVEF) but similarly high mortality [4]. Patients with HFpEF tend to be older, more commonly female, hypertensive, and in contrast to heart failure with reduced ejection fraction (HFrEF), display a low prevalence of coronary artery disease (Table 1) [5]. With the aging population the prevalence of HFpEF will increase; some predict it will surpass HFrEF as the most prevalent heart failure phenotype over the coming years [6]. Yet despite these trends the pathophysiology of HFpEF remains incompletely understood and to date no effective evidence-based therapeutic strategies have emerged.

To begin to unravel complex pathophysiology, a holistic "physiological map" across the spatial domains of cell, tissue and organs may be constructed to highlight the primary components involved in feedback between these domains and their complex multi-directional manner (Figure 1). From an organ-level perspective, important factors identified to contribute to the HFpEF syndrome include concentric left ventricular (LV) remodeling, diastolic and subtle systolic dysfunction, decreased arterial distensibility, and abnormal interaction between LV performance and the systemic arterial system (ventricular-vascular coupling) [7-12]. Each of these measurable factors is likely influenced by processes acting at cellular, subcellular, and molecular levels, for example deranged extracellular matrix turnover [13,14], endothelial dysfunction [15], and activation of inflammatory pathways [16]. Additional factors such as chronotropic incompetence [17-19] and increased sensitivity to neurohormonal stimulation [20] further suggest an orchestrated multifaceted mechanism underlying the development of the HFpEF syndrome. While traditional hypothesis-driven research has been invaluable in delineating individual etiological pathways in heart failure, particularly HFrEF, one could argue that sole use of reductionist lines of investigation may lack the sensitivity to adequately describe the complex and potentially non-linear interactions involved in a heart failure syndrome. Specifically for HFpEF, the challenge remains to identify nuances that lead to the development of clinical symptoms from asymptomatic states pertaining to aging and hypertension, and to explain the marked female preponderance of this condition. In this respect a conceptual approach incorporating 'omics methodologies may not only complement traditional biochemical research but generate heretofore unidentified mechanisms, biomarkers, and therapeutic targets.

A visual representation of current knowledge of factors pertaining to HFpEF, integrated within the overall context of cardiovascular physiology as depicted in Figure 1, may be useful for initiating the design of vasculomic studies. These factors are discussed with supporting evidence in subsequent sections and emphasized in Figure
2. Components represented in the figures are highlighted by italics in the following sections.

\section{From hypertension to $\mathrm{HFpEF}$}

Across epidemiological surveys, hypertension recurrently emerges as the most frequent co-morbidity in patients with HFpEF. Prevalence reports range between 59 and $86 \%$ across large hospitalized and community-based cohorts [21-23], and 60 to $88 \%$ in HFpEF clinical trials [24-27]. While this strong association has provided a useful investigational model for research into $\mathrm{HFpEF}$, the specific etiology of hypertension in these patients is rarely reported. Animal studies to model HFpEF to date have consisted of renovascular hypertension $[20,28]$, yet in humans, hypertension is widely recognized to have multiple etiologies, and further influenced by sex, age, and likely genetic factors. Although human studies incorporate this variation to some extent, it is unknown if such heterogeneity masks potential predictors of HFpEF within unidentified hypertensive subgroups. For example, mechanisms inducing HFpEF in elderly hypertensive females, the most frequently encountered clinical scenario may differ between those developing hypertension under the influence of an estrogenic or anestrogenic state, i.e., pre- and post-menopausal hypertension. Consequently a first-line traditional linear approach to scientific enquiry may limit the rate of knowledge acquisition in this regard, where employing an 'omics-wide lens to genetically or biochemically profile research subjects may enable more robust phenotyping and facilitate more avenues for hypothesis-driven investigation. This concept will be developed further below.

Genomics of hypertension and HFpEF: Gene expression profiling involves the use of microarray platforms which can deliver both qualitative and quantitative information regarding gene function, i.e., identification of gene activation or silencing, and expression levels respectively. The technique has been usefully applied in distinguishing etiology in some HFrEF studies; Kittleson et al. were able to distinguish ischemic from non-ischemic heart failure with $89 \%$ sensitivity and specificity in 48 patients with either end-stage $(n=41)$ or newly diagnosed $(n=7)$ HFrEF [29]. Similarly in both obstructive and nonobstructive hypertrophic cardiomyopathy (HCM), the latter a classic paradigm of HFpEF, genotyping has proved invaluable in establishing pathogenesis. The difficulty with HFpEF is that it nearly always arises in the context of several co-morbid conditions, in particular hypertension and obesity, each of which has its own genetic susceptibility that may additionally influence heart failure risk. Uncovering genetic risk modifiers for an HFpEF phenotype would require construction of elaborate etiological pathways through adequately powered studies, arguably the remit of 'omics style informatics, though the complexity of which should not be underestimated. Taking hypertension as an example of a multifactorial disease and HFpEF antecedent: while candidate gene studies have proposed many potentially contributory single nucleotide polymorphisms (SNPs), results from two large primary genome-wide association studies (GWAS) for hypertension were modest at best [30,31], and only on meta-analyses were genomewide significant associations observed, i.e., significance withstanding correction for multiple testing [32,33]. It is important to note that GWAS findings only indicate approximate loci of causal variant genes. Neighboring alleles must be considered equally or perhaps more significant in determining a particular phenotype until functional mechanisms can be verified. Nevertheless ongoing efforts at large-scale genetic profiling spawning from databases such as the Human Genome [34] and HapMap [35] projects and newer next-generation sequencing 
Citation: Zakeri R, Vallejo-Giraldo C, Taylor KE, Miller VM, Schaible NS (2011) Using Heart Failure with Preserved Ejection Fraction to Understand an 'Omics Approach for Evaluating Vascular Dysfunction and Cardiovascular Disease. J Neurol Neurophysiol S1. doi:10.4172/2155-9562. S1-006

technologies offer the potential to reveal as yet unidentified genetic variants with larger biological effects.

Inflammation, fibrosis \& remodeling: That being said, it is unlikely that the development of HFpEF is principally genetically driven. While the genome represents the initial layer of complexity in both normal and disease pathways, the important 'effector' products of genes are proteins. These proteins may be subject to alternative splicing and posttranslational processing, both of which may independently confer risk of disease. Proteomic technologies permit the examination of global alterations in protein abundance and expression in cardiovascular disease states [36]. Various human cardiac protein databases are already available online, largely based on information from two dimensional polyacrylamide gel electrophoresis (2DPAGE) and/or combined with mass spectrometry data [2,37]. The Human Plasma Proteome Project pilot phase (HUPO PPP) constitutes an impressive international collaborative effort to analyze human plasma samples with proteomic platforms [38]. From a subset of 3020 proteins identified on the basis of two or more peptides on MS/MS experiments, 345 were identified as having cardiovascular-related functions, and a number specifically pertaining to heart failure and ventricular remodeling. Among these, cytokines including transforming growth factor beta (TGF- $\beta$ ) and tumor necrosis factor (TNF) emerged as potential mediators of a heart failure syndrome, confirming previous findings in HFrEF [39]. Although a separate proteomic analysis of HFpEF has not yet been published, there is experimental evidence that proinflammatory pathways and immune activation are involved. When compared to control subjects, Niethammer et al. observed increased levels of soluble TNF receptors 1 and 2 and elevated serum concentrations of pro-inflammatory cytokines IL-6, IL-10 and c-reactive protein (CRP) in patients with HFpEF and hypertension [16]. Levels of all markers remained highest in the HFrEF arm of the study, suggesting either a distinct pathophysiological pathway of systemic immune activation or the possibility that HFpEF, at least in some patients, may progress to an HFrEF phenotype. Whether HFpEF constitutes part of a continuum in heart failure or a distinct entity remains controversial. Furthermore, while both the HUPO PPP and the latter study examined both age-appropriate adult males and females, it remains unclear if immune function varies by sex in this predominantly female-afflicting syndrome, as occurs in autoimmune and connective tissue diseases such as rheumatoid arthritis or lupus. Further large-scale 'omics-style data analyses would enable the adequately powered analyses, also including sex as a biological variable, necessary to answer these questions.

Other proteins proposed to be differentially regulated in HFpEF include those related to extracellular matrix (ECM) remodeling. In murine models of hypertension, inflammatory cells have been colocalized with collagen producing fibroblasts [40]. Collagen is the predominant constituent of the ECM, and increased collagen content is a precursor to fibrosis. It is plausible that an increase in ECM fibrosis may lead to LV diastolic stiffness, as is observed in the HFpEF syndrome. To this end, histological analyses in both human and animal studies have confirmed the presence of an increased myocardial collagen volume fraction in hypertensive and heart failure states [41]. In HFpEF, additional evidence from circulating biomarkers of collagen metabolism points towards an active fibrotic process involving both increased collagen synthesis and decreased degradation [42], though two areas of contention exist. Firstly, in an early and unique study of endomyocardial biopsy tissue from patients with a clinical diagnosis of HFpEF, only two-thirds of the study cohort $(n=12)$ displayed a high collagen volume fraction consistent with fibrosis, despite high resting tension within mechanically isolated cardiomyocytes [41] There were no patients with high collagen volume fraction (fibrosis) and low resting cardiomyocyte tension, suggesting that intrinsic cardiomyocyte stiffness can precede ECM fibrosis in HFpEF. Secondly, it is unclear whether ventricular remodeling is similar for both sexes. In mice models of pressure overloaded myocardium (transverse aortic constriction), used as a model hypertensive state, higher expression of genes pertaining to ECM remodeling was observed [43]. These findings are supported by a human study of aortic constriction where males displayed more disordered collagen architecture [44]. If fibrosis were to underlie the development of HFpEF, this is at variance with the female preponderance seen in epidemiological surveys. An 'omics based study could be designed specifically able to address these questions and tease out possible sex-specific distinctions in HFpEF pathogenesis. Additionally, intrinsic cardiomyocyte stiffness has been linked to expression of a shorter and more stiff isoform of the cytoskeletal protein titin (N2B) [45], its altered phosphorylation, or increased formation of intramolecular disulfide cross-bridges [46-48]. Concurrent large-scale examination of the HFpEF proteome including descriptions of multiple titin and collagen related proteins, within male and female models would offer valuable insights here.

Fibrosis is not restricted to the myocardium; several studies have described the contribution of the ECM to arterial stiffness [49]. This is important since $\mathrm{HFpEF}$ is a cardio-vascular syndrome and it is purported that symptoms in HFpEF, particularly exercise limitation and acute pulmonary edema, are heavily determined by properties of the vasculature [50-52]. Elevated arterial stiffness is observed in aging and hypertension, and is associated with increased myocardial oxygen demand, a concomitant reduction in ventricular compliance, and impaired handling of acute changes in blood pressure or circulating volume [50,53]. For maximal mechanical power transfer, ventricular and vascular (arterial) properties must match (ventricular-vascular coupling). In HFpEF, ventricular stiffness exceeds arterial stiffness (deranged coupling), although importantly the absolute values of both are elevated compared to normotensive controls [51]. In this context, any acute changes in afterload leads to a disproportionate increase in systolic load on the ventricle, thereby increasing LV diastolic filling pressure, and precipitating acute pulmonary edema [50]. Animal models of essential hypertension, such as the spontaneously hypertensive rat (SHR) have enabled investigation into the cellular and molecular determinants of arterial stiffness; though potentially introducing bias through the predominant study of animals of a single sex, i.e. male. Much of the focus, including genotype-phenotype studies, has been on mechanical factors regulating vascular structure (cytoskeletal and cell membrane proteins, ECM proteins e.g. collagens and elastin). However, with the use of microarray gene technology on human aortic tissue, the differential expression of proteoglycan signaling molecules and various transcripts of yet unknown function were revealed to play an equally important role [54]. Little data exists on the association of proteoglycans with arterial stiffness, and certainly any role in HFpEF pathogenesis is currently unknown. Herein lies an example of how genomics data may generate new avenues for proteomic investigation in HFpEF or a potentially novel therapeutic target.

\section{Aging, diastolic dysfunction, and HFpEF}

Normal aging is associated with a degree of increased ventricular stiffness (reduced compliance) and prolongation of diastolic relaxation, known as diastolic dysfunction [55]. In HFpEF, however, such changes 
Citation: Zakeri R, Vallejo-Giraldo C, Taylor KE, Miller VM, Schaible NS (2011) Using Heart Failure with Preserved Ejection Fraction to Understand an 'Omics Approach for Evaluating Vascular Dysfunction and Cardiovascular Disease. J Neurol Neurophysiol S1. doi:10.4172/2155-9562. S1-006

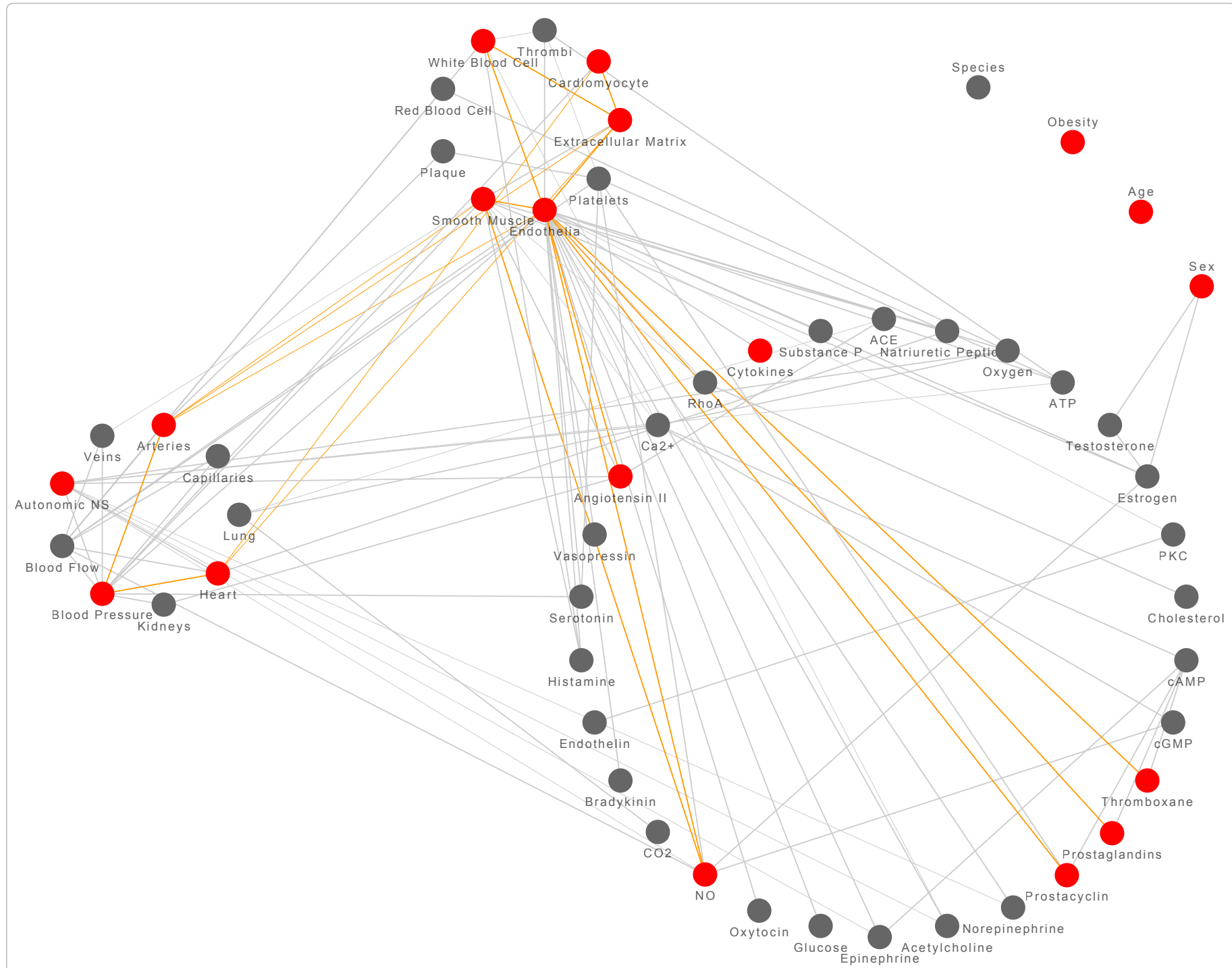

Figure 2: HFpEF vasculomic network. The pathophysiology of HFpEF is highlighted on the same physiological map shown in Figure 1 with the nodes and edges that represent factors relevant to the etiology of HFpEF highlighted in red to integrate current knowledge about factors related to HFpEF into the overall cardiovascular physiology context. This technique may be useful for initiating the design of vasculomic studies. This figure was created by N. Schaible using freely available software, Cytoscape (cytoscape.org), version 2.8.1. [75]

are more pronounced and correlate with hemodynamic alterations and the appearance of heart failure symptoms [56]. Diastolic dysfunction is in fact a central criterion for the diagnosis of HFpEF [8]. The correlation between HFpEF and aging may be partly explained by a greater prevalence of co-morbidities such as hypertension, whereby ECM fibrosis and changes in cardiomyocyte titin expression increase passive ventricular stiffness (see earlier), but may also relate to ageassociated changes in regulation of myocardial relaxation. This component of normal diastole is a metabolically active process [57]. In order to entertain any such hypotheses, and ultimately predict who is likely to develop HFpEF, a detailed understanding of the biochemical and molecular changes that characterize myocardial relaxation within the context of diastolic dysfunction would be necessary. Few studies to date have addressed this. Junhong et al. applied proteomic methods to a rat model of renovascular hypertension induced diastolic dysfunction [28]. Compared to sham operated control rats, diastolic dysfunction was associated with differential protein expression in calcineurin signaling cascades and cellular energetic pathways, thus providing novel targets for further investigation. The authors state that titin isoform and collagen expression were not examined in this study on account of proteomic technology reasons; so their relative contribution remains unknown. Furthermore, it must be emphasized that proteomic data is only suggestive of an association to a particular physiological state until there has been validation of clinical significance [28]. This highlights an important issue. The application of 'omic technologies to cardiovascular disease most often requires cardiac tissue which is infrequently obtained in routine clinical care. Animal models are therefore heavily relied upon; the importance of the precise model utilized must not be underestimated. In the aforementioned study the calcineurin pathway was implicated in the pathogenic proteome of renal-clipped hypertensive male rats with diastolic dysfunction. Overactivated calcineurin signaling has similarly been identified in other models of increased LV afterload (aortic constriction) [58,59], but not observed in studies utilizing spontaneously hypertensive rats 
Citation: Zakeri R, Vallejo-Giraldo C, Taylor KE, Miller VM, Schaible NS (2011) Using Heart Failure with Preserved Ejection Fraction to Understand an 'Omics Approach for Evaluating Vascular Dysfunction and Cardiovascular Disease. J Neurol Neurophysiol S1. doi:10.4172/2155-9562. S1-006

Page 6 of 10

(SHR), where activation of other signaling pathways such as those involving NF-kappa B have rather been observed [58,60]. HFpEF is arguably a complex multifactorial disease state, and individually identified etiological pathways may only pertain to the particular experimental model used. Furthermore, each individual 'model' is likely to be an imperfect representation of this complexity: Junhong et al utilized only male hypertensive rats. It is not clear whether this would adequately represent the clinical, biochemical, and molecular processes underlying HFpEF in females; which is an important consideration given the markedly female predominance of this disease.

Additionally a key corollary of the application of global 'omicstype' investigations is the generation of large amounts of data from differing sources. The subsequent task remains to model functional networks based on this data that are broadly capable of representing all components of the 'system' or disease within the environment and contexts in which it occurs. For this, a combination of informatics and traditional hypothesis-based lines of investigation will prove essential.

\section{Endothelial dysfunction and impaired vasodilator response}

The definition of HFpEF extends beyond myocardial diastolic dysfunction and involves maladaptive vascular remodeling and function [12]. The contribution of arterial stiffness within the central vasculature has been outlined above, but another proposed contributing factor to the pathophysiology of HFpEF is dysfunction of the vascular endothelium. The endothelium releases endotheliumderived relaxing factors (EDRFs) such as nitric oxide (NO) and prostacyclin, which decrease vascular tone, as well as factors collectively known as endothelium derived contracting factors (EDCFs) including cyclo-oxygenase (COX) derived metabolites of the arachidonic acid (AA) cascade and thromboxane A2 (TXA2), which increase vascular tone. Both COX derived metabolites of the AA cascade and TXA2 are pro-inflammatory factors, suggesting an association between inflammation, endothelial dysfunction, and vascular tone. Control of the balance between EDRFs and EDCFs is important. In healthy endothelium EDRFs predominate and counteract the constrictive effects of EDCFs. In systemic hypertension this balance is shifted, there is decreased bioavailability of endothelial-derived $\mathrm{NO}$, and relatively unopposed activity of EDCFs [61], the degree of which predicts the severity of future vascular complications [62]. Thus the endothelium is able to act as both a source and effector of inflammation and both of these negative and positive feedback mechanisms must be considered in models which include the endothelium in the etiology of the disease.

Evidence of a shift in the balance of dilatory to constrictor functions of the endothelium in the etiology of HFpEF is derived from measures of reactive hyperemia following occlusion of forearm blood flow [12]. Increases in blood flow of the brachial artery after release of the cuff occlusion (reactive hyperemia index, RHI) were less in HFpEF and hypertensive patients compared with normotensive

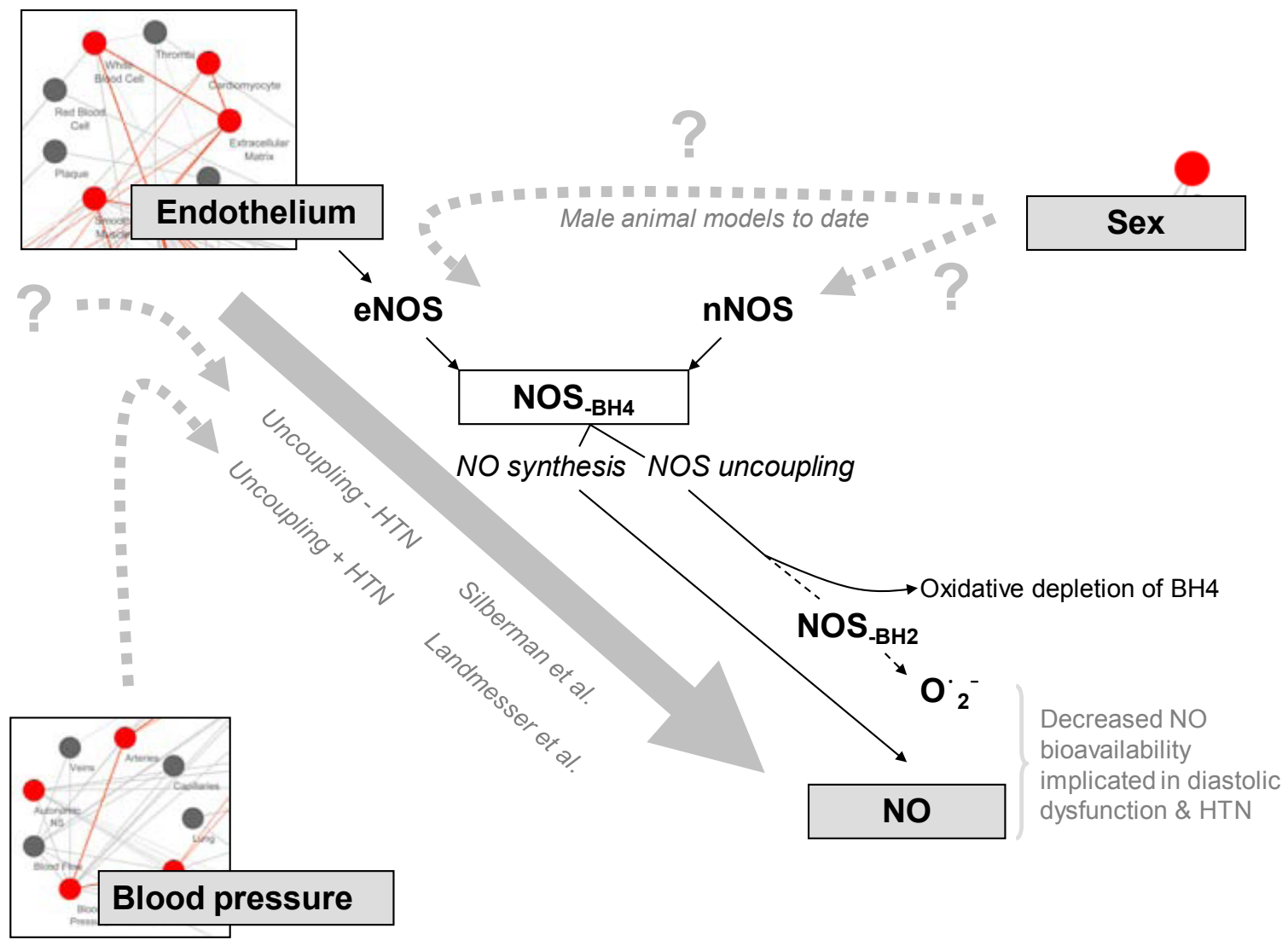

Figure 3: Relationship between endothelial cells and nitric oxide (NO) synthesis, as implicated in HFpEF pathophysiology. Pathways shown represent experimental evidence to date; avenues for future experimental investigation are highlighted by '?'. Abbreviations: NO, nitric oxide; eNOS, NOS, nitric oxide synthase, eNOS, endothelial derived nitric oxide synthase; nNOS, neuronal nitric oxide synthase; $\mathrm{BH}^{4}$, tetrahydrobiopterin (NOS cofactor); ${ }^{\circ} 2^{-}$, superoxide; $\mathrm{HTN}^{2}$, hypertension. Landmesser et al. [63], Silberman et al. [64]. 
Citation: Zakeri R, Vallejo-Giraldo C, Taylor KE, Miller VM, Schaible NS (2011) Using Heart Failure with Preserved Ejection Fraction to Understand an 'Omics Approach for Evaluating Vascular Dysfunction and Cardiovascular Disease. J Neurol Neurophysiol S1. doi:10.4172/2155-9562. S1-006

control subjects, suggesting a decrement in endothelium dependent vasodilatation, i.e., endothelial dysfunction. A proposed mechanism is uncoupling of nitric oxide synthase (NOS), the enzyme catalyzing NO synthesis, which has been documented in the setting of hypertension and renin-angiotensin system (RAS) activation [63]. Interestingly though, not all patients with HFpEF in this study were reported to have preceding or concurrent hypertension. Taking this point further, Silberman et al. demonstrated that NOS uncoupling and oxidative depletion of its cofactor tetrahydrobiopterin (BH4), was implicated in the pathogenesis of diastolic dysfunction, in male mice in the absence of hypertension. And further that oral therapy with the depleted cofactor was able to reverse this diastolic dysfunction [64]. Whether these observations apply to female animals is unclear. Recognizing that NOS may be of both endothelial (eNOS) and neuronal (nNOS) origin; there is nonetheless a suggestion of a link between an endotheliumderived and regulated product and diastolic dysfunction, independent of hypertension.

By incorporating these experimental data within a physiological map representing the known components of the HFpEF syndrome (Figure 2), several avenues for future experimental work emerge (Figure 3). For instance, a potential application of 'omics investigations here could be to comprehensively characterize endothelial gene and protein expression within HFpEF cohorts. Certain polymorphisms in the NO system have been suggested to have greater effects in women than in men $[65,66]$; an 'omics analysis may offer revealing insights into sex-specific contributions of endothelial or $\mathrm{NO}$ function in HFpEF pathogenesis. Furthermore, in contrast to an 'omics approach that requires traditional animal and human experimental models to provide clinical validation here it may be possible to identify a unique genotype-phenotype combination, particularly HFpEF patients without hypertension, in whom the endothelium may present a genuine therapeutic target. Hence the 'omics approach may facilitate the goal of individualized medicine.

\section{Impaired chronotropic reserve}

The 'omics approach investigates networks of genes, proteins and molecules interacting under counter-regulatory signals to achieve homeostasis. These signals may originate from more than one regulatory system, for example in the case of cardiac output and blood pressure regulation the autonomic nervous system plays an integral role. In diseased states, this balance is disrupted. HFpEF patients demonstrate autonomic dysfunction [17]. Compared to age and gender-matched healthy controls and hypertensive patients, HFpEF patients were shown to exhibit chronotropic incompetence, i.e. inadequate elevation of heart rate at peak exercise, and prolonged heart rate recovery time following exercise $[17,19]$. These phenomena reflect the balance between parasympathetic and sympathetic activation and withdrawal at various stages of exercise, and contribute to symptoms in HFpEF. What is unclear from existing studies is whether this altered regulation is an adaptive response to prolong diastolic filling time, or maladaptive. Furthermore, it is difficult to account for the female predominance of HFpEF from these generalized data as little is known regarding the control of vascular resistance in women. A linear relationship between total peripheral resistance and sympathetic nerve activity does not exist for young women as for men [67] but it is not known if these relationships change with age or hormonal status in women as they age. While some data suggest that there is a withdrawal of vagal tone in women with aging, most data on heart rate variability (turbulence) is not analyzed by sex [68,69]. A detailed analysis at the proteomic and metabolomic level of patients with differing degrees of diastolic dysfunction and symptomatic stages (from asymptomatic hypertension to HFpEF) considering sex and hormonal status may facilitate understanding in this regard.

\section{Integrating cardiovascular disease data into an 'omics framework}

The essence of an 'omics study consists of finding meaningful differences in data filled with many sources of variation. A simple example would be the comparison of genomic data to identify genetic markers for disease. With the exception of monogenetic diseases with Mendelian patterns of inheritance, it is rare for a disease to be solely genetically determined, and many sources and forms of data must be considered simultaneously in order to find meaningful differences, i.e, those that have biomedical significance as opposed to a difference due to confounding or bias. Importantly, once data is collected, differences all appear in the same way. For this reason, the precise design of an 'omics study is extremely important. What distinguishes a meaningful difference from noise requires application of differing expertise working together as an interdisciplinary team. Such a team could include physiologists, biochemists, pharmacologists, molecular scientists, and geneticists, along with clinician scientists, clinicians, statisticians and epidemiologists, depending on the disease under investigation. Each offers a unique skill set and viewpoint necessary for valid and effective scientific research. An important consideration is that collaboration should be present from the initial phase of study design and continue to provide the adequate care and scrutiny required throughout the entire study process [70]. It is the role of scientists and clinicians to frame a robust and objective initial study idea by identifying both the final quantity of interest (endpoint) and potential confounding factors, integrating physiological and clinical data. The aforementioned discussion on HFpEF is an example of how raw knowledge may be used to synthesize the initial study design; the creation of a "pathophysiological map" may help in this regard (Figure 1 and Figure 2). It is also important to frame this knowledge in a way that fosters subsequent interdisciplinary collaboration.

Limitations of physiological maps and 'omics approaches: Physiological maps, such as those illustrated in (Figure 1 and Figure 2) can serve as an initial step towards constructing hypotheses and describing the relevant pathophysiology of a disease but are necessarily oversimplified for the purposes of visual representation. As further knowledge is assumed, a map may be expanded to some degree, although input-output processing within molecular domains and details of biochemical pathways may still be difficult to display. Moreover, it must be recognized that, particularly in the case of HFpEF, the important contributions of multiple time points (cyclic positive and negative-feedback) even within an age group (to include reproductive or hormonal status) are not readily displayed. Nonetheless, where such maps are useful is in highlighting the complex interconnectedness between components (each represented as a node) involved in cardiovascular physiology and those relevant to consider in cardiovascular disease. Nodes which are more heavily connected than others, for example endothelial cells, can be readily identified as key effectors of cardiovascular control and therefore potential mediators of disease or targets for therapy. These visualizations also help to identify gaps in current knowledge that could lead to new insights into disease etiology or therapeutic targets. 
Citation: Zakeri R, Vallejo-Giraldo C, Taylor KE, Miller VM, Schaible NS (2011) Using Heart Failure with Preserved Ejection Fraction to Understand an 'Omics Approach for Evaluating Vascular Dysfunction and Cardiovascular Disease. J Neurol Neurophysiol S1. doi:10.4172/2155-9562. S1-006

Page 8 of 10

Data used to construct a physiological map may be derived from varying sources and this represents a potential source of bias to consider both in the analysis of a map and the design of an 'omics study. The variety of experimental models and populations used to study the pathophysiology of HFpEF has been outlined in the preceding discussion. However, it must be appreciated that outcomes observed relating to specific species, strains of animals or sex are not categorically generalizable, and that validation in alternative or more representative populations may still be required. The absence of data from female animals and/or cells (unpublished survey conducted by our group) is striking and represents opportunities to explore the physiological basis of a disease that presents with a female predominance [71-74]. There are potential confounders within HFpEF pathophysiology, such as obesity, which are proposed to have multi-level interactions and implicate multiple other significant components already mentioned. There are also ubiquitous processes germane to cardiovascular physiology and disease such as intracellular calcium signaling. For simplicity we have elected not to discuss these as separate components within this review.

Finally, certain nodes within a map may exhibit heterogeneity, and it may be misleading to represent, for example, all endothelial cells as a single node. The reality is that endothelial cells exhibit a considerable degree of heterogeneity conferred in part by anatomical location and environment as well as the specific complement of adrenoreceptors ( $\alpha$ and $\beta$ subtypes). To acknowledge components where heterogeneity is present is an important consideration not only to HFpEF pathogenesis but to vascular physiology as a whole. All possible interactions are mapped to the heterogeneous node with the caveat that only a subset may actually be valid for any given endothelial cell to follow the previous example. The presence of heterogeneity is a potentially confounding influence in vasculomics studies which probably deserves more attention and detail than a two dimensional network can illustrate.

\section{Conclusion}

The introduction and availability of novel 'omics technologies into the scientific arena offers an alternative and complementary paradigm to traditional hypothesis-driven research. It is now possible to comprehensively describe pathophysiology at a genetic, proteomic and molecular level, and generate elaborate network maps that more closely represent the truly integrated nature of cardiovascular disease. Ultimately the goal of this holistic approach is to generate novel and true insights into disease pathogenesis, in order to propose new diagnostic or therapeutic strategies. Heart failure with preserved ejection fraction is an example of an increasingly prevalent disease where there is a desperate need for a better understanding of etiology, to include mechanisms underlying its female predominance, and potential targets for therapy. Early efforts have been made to delineate hemodynamic and cellular pathophysiologic aberrations in HFpEF antecedents such as hypertension and ventricular diastolic dysfunction, for which 'omics technologies have initially proved meritorious. Importantly though, complexities and confounding factors are key considerations in the design of 'omics studies. These complexities and confounding factors include techniques of data collection and generalizability of those data. The development of 'omics-techniques have followed the demand for more individualized and advanced therapies in an aging population with increasing co-morbidities. The true challenge of the 'omics era will be in the appropriate design, handling, and interpretation of multidimensional data in order to meet this goal.

\section{Acknowledgement}

This work was funded in part by the Mayo Clinic Graduate School of Medicine.

\section{References}

1. Evans GA (2000) Designer science and the "omic" revolution. Nat Biotechnol 18: 127.

2. Ruegg C, Tissot JD, Farmer P, Mariotti A (2008) Omics meets hypothesisdriven research. Partnership for innovative discoveries in vascular biology and angiogenesis. Thromb Haemost 100: 738-746.

3. Hunt SA, Abraham WT, Chin MH, Feldman AM, Francis GS, et al. (2009) 2009 Focused update incorporated into the ACC/AHA 2005 Guidelines for the Diagnosis and Management of Heart Failure in Adults A Report of the American College of Cardiology Foundation/American Heart Association Task Force on Practice Guidelines Developed in Collaboration With the International Society for Heart and Lung Transplantation. J Am Coll Cardiol 53: e1-e90.

4. Owan TE, Hodge DO, Herges RM, Jacobsen SJ, Roger VL, et al. (2006) Trends in prevalence and outcome of heart failure with preserved ejection fraction. N Engl J Med 355: 251-259.

5. Bhatia RS, Tu JV, Lee DS, Austin PC, Fang J, et al. (2006) Outcome of heart failure with preserved ejection fraction in a population-based study. $\mathrm{N}$ Engl $J$ Med 355: 260-269.

6. Borlaug BA, Paulus WJ (2011) Heart failure with preserved ejection fraction pathophysiology, diagnosis, and treatment. Eur Heart J 32: 670-679.

7. Zile MR, Baicu CF, Gaasch WH (2004) Diastolic heart failure--abnormalities in active relaxation and passive stiffness of the left ventricle. N Engl J Med 350 1953-1959.

8. Paulus WJ, Tschope C, Sanderson JE, Rusconi C, Flachskampf FA, et al (2007) How to diagnose diastolic heart failure: a consensus statement on the diagnosis of heart failure with normal left ventricular ejection fraction by the Heart Failure and Echocardiography Associations of the European Society of Cardiology. Eur Heart J 28: 2539-2550.

9. Borlaug BA, Lam CS, Roger VL, Rodeheffer RJ, Redfield MM (2009) Contractility and ventricular systolic stiffening in hypertensive heart disease insights into the pathogenesis of heart failure with preserved ejection fraction. J Am Coll Cardiol 54: 410-418.

10. Phan TT, Abozguia K, Nallur Shivu G, Mahadevan G, Ahmed I, et al. (2009) Heart failure with preserved ejection fraction is characterized by dynamic impairment of active relaxation and contraction of the left ventricle on exercise and associated with myocardial energy deficiency. J Am Coll Cardiol 54: 402409

11. Tan YT, Wenzelburger F, Lee E, Heatlie G, Leyva F, et al. (2009) The pathophysiology of heart failure with normal ejection fraction: exercise echocardiography reveals complex abnormalities of both systolic and diastolic ventricular function involving torsion, untwist, and longitudinal motion. J Am Coll Cardiol 54: 36-46.

12. Borlaug BA, Olson TP, Lam CS, Flood KS, Lerman A, et al. (2010) Global cardiovascular reserve dysfunction in heart failure with preserved ejection fraction. J Am Coll Cardiol 56: 845-854.

13. Ahmed SH, Clark LL, Pennington WR, Webb CS, Bonnema DD, et al. (2006) Matrix metalloproteinases/tissue inhibitors of metalloproteinases: relationship between changes in proteolytic determinants of matrix composition and structural, functional, and clinical manifestations of hypertensive heart disease. Circulation 113: 2089-2096.

14. Gonzalez A, Lopez B, Querejeta R, Zubillaga E, Echeverria T, et al. (2010) Filling pressures and collagen metabolism in hypertensive patients with heart failure and normal ejection fraction. Hypertension 55: 1418-1424.

15. Tschope C, Bock CT, Kasner M, Noutsias M, Westermann D, et al. (2005) High prevalence of cardiac parvovirus B19 infection in patients with isolated left ventricular diastolic dysfunction. Circulation 111: 879-886.

16. Niethammer M, Sieber M, von Haehling S, Anker SD, Munzel T, et al. (2008) Inflammatory pathways in patients with heart failure and preserved ejection fraction. Int J Cardiol 129: 111-117.

17. Borlaug BA, Melenovsky V, Russell SD, Kessler K, Pacak K, et al. (2006) Impaired chronotropic and vasodilator reserves limit exercise capacity in patients with heart failure and a preserved ejection fraction. Circulation 114 2138-2147. 
Citation: Zakeri R, Vallejo-Giraldo C, Taylor KE, Miller VM, Schaible NS (2011) Using Heart Failure with Preserved Ejection Fraction to Understand an 'Omics Approach for Evaluating Vascular Dysfunction and Cardiovascular Disease. J Neurol Neurophysiol S1. doi:10.4172/2155-9562. S1-006

18. Brubaker PH, Joo KC, Stewart KP, Fray B, Moore B, et al. (2006) Chronotropic incompetence and its contribution to exercise intolerance in older heart failure patients. J Cardiopulm Rehabil 26: 86-89.

19. Phan TT, Shivu GN, Abozguia K, Davies C, Nassimizadeh M, et al. (2010) Impaired heart rate recovery and chronotropic incompetence in patients with heart failure with preserved ejection fraction. Circ Heart Fail 3: 29-34.

20. Mohammed SF, Ohtani T, Korinek J, Lam CS, Larsen K, et al. (2010) Mineralocorticoid accelerates transition to heart failure with preserved ejection fraction via "nongenomic effects". Circulation 122: 370-378

21. Bursi F, Weston SA, Redfield MM, Jacobsen SJ, Pakhomov S, et al. (2006) Systolic and diastolic heart failure in the community. J Am Med Assoc 296: 2209-2216.

22. Masoudi FA, Havranek EP, Smith G, Fish RH, Steiner JF, et al. (2003) Gender age, and heart failure with preserved left ventricular systolic function. J Am Coll Cardiol 41: 217-223.

23. Lenzen MJ, Scholte op Reimer WJ, Boersma E, Vantrimpont PJ, Follath F, et al. (2004) Differences between patients with a preserved and a depressed left ventricular function: a report from the EuroHeart Failure Survey. Eur Heart J 25: $1214-1220$.

24. McMurray JJ, Carson PE, Komajda M, McKelvie R, Zile MR, et al. (2008) Heart failure with preserved ejection fraction: clinical characteristics of 4133 patients enrolled in the I-PRESERVE trial. Eur J Heart Fail 10: 149-156.

25. Ahmed A, Rich MW, Fleg JL, Zile MR, Young JB, et al. (2006) Effects of digoxin on morbidity and mortality in diastolic heart failure: the ancillary digitalis investigation group trial. Circulation 114: 397-403.

26. Yusuf S, Pfeffer MA, Swedberg K, Granger CB, Held P, et al. (2003) Effects of candesartan in patients with chronic heart failure and preserved left-ventricular ejection fraction: the CHARM-Preserved Trial. Lancet 362: 777-781.

27. Cleland JG, Tendera M, Adamus J, Freemantle N, Polonski L, et al. (2006) The perindopril in elderly people with chronic heart failure (PEP-CHF) study. Eur Heart J 27: 2338-2345.

28. Junhong W, Jing Y, Jizheng M, Shushu Z, Xiangjian C, et al. (2008) Proteomic analysis of left ventricular diastolic dysfunction hearts in renovascular hypertensive rats. Int J Cardiol 127: 198-207

29. Kittleson MM, Ye SQ, Irizarry RA, Minhas KM, Edness G, et al. (2004) Identification of a gene expression profile that differentiates between ischemic and nonischemic cardiomyopathy. Circulation 110: 3444-3451.

30. Wellcome Trust Case Control Consortium (2007) Genome-wide association study of 14,000 cases of seven common diseases and 3,000 shared controls. Nature 447: 661-678.

31. Levy D, Larson MG, Benjamin EJ, Newton-Cheh C, Wang TJ, et al. (2007) Framingham Heart Study 100K Project: genome-wide associations for blood pressure and arterial stiffness. BMC Med Genet 8 Suppl 1: S3.

32. Newton-Cheh C, Johnson T, Gateva V, Tobin MD, Bochud M, et al. (2009) Genome-wide association study identifies eight loci associated with blood pressure. Nat Genet 41: 666-676.

33. Levy D, Ehret GB, Rice K, Verwoert GC, Launer LJ, et al. (2009) Genome-wide association study of blood pressure and hypertension. Nat Genet 41: 677-687.

34. Lander ES, Linton LM, Birren B, Nusbaum C, Zody MC, et al. (2001) Initia sequencing and analysis of the human genome. Nature 409: 860-921.

35. Frazer KA, Ballinger DG, Cox DR, Hinds DA, Stuve LL, et al. (2007) A second generation human haplotype map of over 3.1 million SNPs. Nature 449: 851 861.

36. Loscalzo J (2003) Proteomics in cardiovascular biology and medicine. Circulation 108: 380-383.

37. http://world-2dpage.expasy.org/list/

38. Berhane BT, Zong C, Liem DA, Huang A, Le S, et al. (2005) Cardiovascularrelated proteins identified in human plasma by the HUPO Plasma Proteome Project pilot phase. Proteomics 5: 3520-3530.

39. Rauchhaus M, Doehner W, Francis DP, Davos C, Kemp M, et al. (2000) Plasma cytokine parameters and mortality in patients with chronic heart failure. Circulation 102: 3060-3067.
40. Hinglais N, Heudes D, Nicoletti A, Mandet C, Laurent M, et al. (1994) Colocalization of myocardial fibrosis and inflammatory cells in rats. Lab Invest 70: 286-294.

41. Borbely A, van der Velden J, Papp Z, Bronzwaer JG, Edes I, et al. (2005) Cardiomyocyte stiffness in diastolic heart failure. Circulation 111: 774-781.

42. Martos R, Baugh J, Ledwidge M, O'Loughlin C, Conlon C, et al. (2007) Diastolic heart failure: evidence of increased myocardial collagen turnover linked to diastolic dysfunction. Circulation 115: 888-895.

43. Witt $\mathrm{H}$, Schubert $\mathrm{C}$, Jaekel J, Fliegner D, Penkalla A, et al. (2008) Sex-specific pathways in early cardiac response to pressure overload in mice. $\mathrm{J}$ Mol Med (Berl) 86: 1013-1024

44. Villari B, Campbell SE, Schneider J, Vassalli G, Chiariello M, et al. (1995) Sex-dependent differences in left ventricular function and structure in chronic pressure overload. Eur Heart J 16: 1410-1419.

45. Nagueh SF, Shah G, Wu Y, Torre-Amione G, King NM, et al. (2004) Altered titin expression, myocardial stiffness, and left ventricular function in patients with dilated cardiomyopathy. Circulation 110: 155-162.

46. Kruger M, Kotter S, Grutzner A, Lang P, Andresen C, et al. (2009) Protein kinase $\mathrm{G}$ modulates human myocardial passive stiffness by phosphorylation of the titin springs. Circ Res 104: 87-94.

47. Borbely A, Falcao-Pires I, van Heerebeek L, Hamdani N, Edes I, et al. (2009) Hypophosphorylation of the Stiff N2B titin isoform raises cardiomyocyte resting tension in failing human myocardium. Circ Res 104: 780-786

48. Hidalgo C, Hudson B, Bogomolovas J, Zhu Y, Anderson B, et al. (2009) PKC phosphorylation of titin's PEVK element: a novel and conserved pathway for modulating myocardial stiffness. Circ Res 105: 631-638.

49. riones AM, Arribas SM, Salaices M (2010) Role of extracellular matrix in vascular remodeling of hypertension. Curr Opin Nephrol Hypertens 19: 187 194

50. Kawaguchi M, Hay I, Fetics B, Kass DA (2003) Combined ventricular systolic and arterial stiffening in patients with heart failure and preserved ejection fraction: implications for systolic and diastolic reserve limitations. Circulation 107: 714-720.

51. Borlaug BA, Kass DA (2008) Ventricular-vascular interaction in heart failure Heart Fail Clin 4: 23-36.

52. Hundley WG, Kitzman DW, Morgan TM, Hamilton CA, Darty SN, et al. (2001) Cardiac cycle-dependent changes in aortic area and distensibility are reduced in older patients with isolated diastolic heart failure and correlate with exercise intolerance. J Am Coll Cardiol 38: 796-802.

53. Kelly RP, Tunin R, Kass DA (1992) Effect of reduced aortic compliance on cardiac efficiency and contractile function of in situ canine left ventricle. Circ Res 71: 490-502.

54. Durier S, Fassot C, Laurent S, Boutouyrie P, Couetil JP, et al. (2003) Physiological genomics of human arteries: quantitative relationship between gene expression and arterial stiffness. Circulation 108: 1845-1851.

55. Mantero A, Gentile F, Gualtierotti C, Azzollini M, Barbier P, et al. (1995) Left ventricular diastolic parameters in 288 normal subjects from 20 to 80 years old. Eur Heart J 16: 94-105.

56. Lam CS, Roger VL, Rodeheffer RJ, Bursi F, Borlaug BA, et al. (2007) Cardiac structure and ventricular-vascular function in persons with heart failure and preserved ejection fraction from Olmsted County, Minnesota. Circulation 115 1982-1990.

57. Stuyvers BD, Miura M, ter Keurs HE (1997) Dynamics of viscoelastic properties of rat cardiac sarcomeres during the diastolic interval: involvement of $\mathrm{Ca}^{2+}$. $\mathrm{J}$ Physiol 502: 661-677.

58. Kokubo M, Uemura A, Matsubara T, Murohara T (2005) Noninvasive evaluation of the time course of change in cardiac function in spontaneously hypertensive rats by echocardiography. Hypertens Res 28: 601-609.

59. Molkentin JD (2000) Calcineurin and beyond: cardiac hypertrophic signaling Circ Res 87: 731-738.

60. Gallego-Delgado J, Lazaro A, Osende JI, Esteban V, Barderas MG, et al (2006) Proteomic analysis of early left ventricular hypertrophy secondary to hypertension: modulation by antihypertensive therapies. J Am Soc Nephrol 17 : S159-164. 
Citation: Zakeri R, Vallejo-Giraldo C, Taylor KE, Miller VM, Schaible NS (2011) Using Heart Failure with Preserved Ejection Fraction to Understand an 'Omics Approach for Evaluating Vascular Dysfunction and Cardiovascular Disease. J Neurol Neurophysiol S1. doi:10.4172/2155-9562. S1-006

Page 10 of 10

61. Wong WT, Wong SL, Tian XY, Huang Y (2010) Endothelial dysfunction: the common consequence in diabetes and hypertension. J Cardiovasc Pharmaco 55: $300-307$

62. Thomas SR, Witting PK, Drummond GR (2008) Redox control of endothelia function and dysfunction: molecular mechanisms and therapeutic opportunities. Antioxid Redox Signal 10: 1713-1765.

63. Landmesser U, Dikalov S, Price SR, McCann L, Fukai T, et al. (2003) Oxidation of tetrahydrobiopterin leads to uncoupling of endothelial cell nitric oxide synthase in hypertension. J Clin Invest 111: 1201-1209.

64. Silberman GA, Fan TH, Liu H, Jiao Z, Xiao HD, et al. (2010) Uncoupled cardiac nitric oxide synthase mediates diastolic dysfunction. Circulation 121: 519-528.

65. Regitz-Zagrosek V, Brokat S, Tschope C (2007) Role of gender in heart failure with normal left ventricular ejection fraction. Prog Cardiovasc Dis 49: 241-251.

66. Laule M, Meisel C, Prauka I, Cascorbi I, Malzahn U, et al. (2003) Interaction of CA repeat polymorphism of the endothelial nitric oxide synthase and hyperhomocysteinemia in acute coronary syndromes: evidence of genderspecific differences. J Mol Med (Berl) 81: 305-309.

67. Hart EC, Charkoudian N, Wallin GG, Curry TB, Eisenach JH, et al. (2009) Sex Differences in Sympathetic Neural-Hemodynamic Balance: implications for human blood pressure regulation. Hypertension 53: 571-576.

68. Stein PK, Barzilay JI (2011) Relationship of Abnormal Heart Rate Turbulence and Elevated CRP to Cardiac Mortality in Low, Intermediate, and High-Risk Older Adults. J Cardiovasc Electrophysiol 22: 122-127.
69. Bauer A, Malik M, Schmidt G, Barthel P, Bonnemeier H, et al. (2008) Heart rate turbulence: standards of measurement, physiological interpretation, and clinical use: International Society for Holter and Noninvasive Electrophysiology Consensus. J Am Coll Cardiol 52: 1353-1365.

70. Von Frese J, Speed T (2010) Designing Rigorous Omics Studies for Biomarker Discovery and Development of Prognostic and Predictive Molecular Diagnostics. 17th International Molecular Medicine Tri-Conference, San Francisco, CA

71. Zucker I, Beery A (2010) Males still dominate animal studies. Nature 465: 690

72. Beery AK, Zucker I (2011) Sex bias in neuroscience and biomedical research. Neurosci Biobehav Rev 35: 565-572.

73. Geller SE, Koch A, Pellettieri B, Carnes M (2011) Inclusion, Analysis and Reporting of Sex and Race/Ethnicity in Clinical Trials: Have we made progress? J Women's Health 20: 315-320.

74. Foulkes MA (2011) After inclusion, information and inference: reporting on clinical trials results after 15 years of monitoring inclusion of women. J Women's Health 20: 829-836.

75. Shannon P, Markiel A, Ozier O, Baliga NS, Wang JT, et al. (2003) Cytoscape: a software environment for integrated models of biomolecular interaction networks. Genome Res 13: 2498-2504.

76. O'Rourke ST, Vanhoutte PM, Miller VM (2006) Vascular Pharmacology. Vascular medicine: a companion to Braunwald's heart disease. Saunders Elsevier, Philadelphia, PA. 